\title{
Ethyl-pyruvate reduces lung injury matrix metalloproteinases and cytokines and improves survival in experimental model of severe acute pancreatitis ${ }^{1}$
}

\author{
Jacques Matone ${ }^{I}$, Ana Iochabel Soares MorettiII, Franz Robert Apodaca-Torrez ${ }^{\mathrm{III}}$, Alberto Goldenberg ${ }^{\mathrm{IV}}$ \\ ${ }^{\mathrm{I}} \mathrm{PhD}$, Postgraduate Program in Gastrointestinal Surgery, Federal University of Sao Paulo - Paulista Medical School (UNIFESP-EPM), Sao Paulo, \\ Brazil. Conception and design of the study, acquisition and interpretation of data, surgical procedures, manuscript preparation, English language. \\ ${ }^{I I} \mathrm{PhD}$, Post-doctoral Fellow, Department of Emergency Medicine, University of Sao Paulo (USP), Brazil. Acquisition and interpretation of data, \\ technical procedures, manuscript preparation. \\ IIIPhD, Assistant Professor, Department of Surgery, UNIFESP-EPM, Sao Paulo, Brazil. Design of the study, critical revision. \\ ${ }^{\text {IV }} \mathrm{PhD}$, Associate Professor, Department of Surgery, UNIFESP-EPM, Sao Paulo, Brazil. Responsible for substantive scientific and intellectual \\ contributions to the study, manuscript writing, critical revision.
}

\section{ABSTRACT}

PURPOSE: To investigate if the ethyl-pyruvate solution could reduce mortality in AP and/or diminish the acute lung injury.

METHODS: Forty male rats, weighing between 270 to 330 grams were operated. An experimental model of severe AP by injection of $0.1 \mathrm{ml} / 100 \mathrm{~g}$ of $2.5 \%$ sodium taurocholate into the bilio-pancreatic duct was utilized. The rats were divided into two groups of ten animals each: CT - control (treatment with $50 \mathrm{ml} / \mathrm{kg}$ of Ringer's solution, intraperitoneal) and EP (treatment with $50 \mathrm{ml} / \mathrm{kg}$ of Ringer ethyl- pyruvate solution, intra-peritoneal), three hours following AP induction. After six hours, a new infusion of the treatment solution was performed in each group. Two hours later, the animals were killed and the pulmonary parenchyma was resected for biomolecular analysis, consisting of: interleukin, myeloperoxidase, MDA, nitric oxide, metalloproteinases and heat shock protein. In the second part of the experiment, another, 20 rats were randomly divided into EP and CT groups, in order to evaluate a survival comparison between the two groups.

RESULTS: There were no significant differences in IL-1B,IL-10, MMP-9, HSP70, nitric oxide, MPO, MDA (lipidic peroxidation) concerning both groups. The levels of IL-6 were significantly diminished in the EP group. Furthermore, the MMP-2 levels were also reduced in the EP group $(\mathrm{p}<0.05)$. The animals from the EP treatment groups had improved survival, when compared to control group $(\mathrm{p}<0.05)$.

CONCLUSION: The ethyl-pyruvate diminishes acute lung injury inflammatory response in acute pancreatitis and ameliorates survival when compared to control group, in the experimental model of necrotizing acute pancreatitis.

Key words: Pancreatitis, Acute Necrotizing. Pyruvic Acid. Inflammation. Rats. 


\section{Introduction}

Mild acute pancreatitis has a low mortality rate, but patients with severe acute pancreatitis (SAP) are more likely to have complications and a much higher death rate. Severe pancreatic injury occurs in $20 \%$ of the patients, and $15 \%$ to $25 \%$ of these patients will end in fatality ${ }^{1,2}$. The amplifying effects of inflammatory and oxidative impairment often lead to SAP-induced complications, which are often regarded as hallmarks of SAP and herald a noted poor outcome. Since respiratory dysfunction is the main cause of death in patients with SAP, elucidating the critical period of acute pancreatitis-associated lung injury (APALI) is clinically important. Even though the pathogenesis of APALI has been studied worldwide in the past years, efforts of researchers mainly focused on inflammatory factors ${ }^{3}$.

Current studies have shown that some inflammatory mediators play an important role in SAP complicated with multiple organ injury ${ }^{4-6}$. This suggests an intricate balance between localized tissue damage with proinflammatory cytokine production and a systemic, anti-inflammatory accelerator of inappropriate migration of proinflammatory agents into the blood stream. The main critical players of this interaction include the pro-inflammatory cytokines IL-1, TNF-alpha, IL-6, IL-8 and metalloproteinases, among others.

Matrix metalloproteinases (MMP) act in processing matrix proteins, cytokines, growth factors, and adhesion molecules. In addition, it has been reported in the literature that MMPs play a role in cell signaling ${ }^{7}$. Matrix metalloproteinases 2 and 9 are very important mediators in tissue inflammatory response and have also been implicated in acute lung injury ${ }^{8-10}$.

The initial management of SAP is supportive based on fluid resuscitation, analgesia, enteral nutrition and thromboprophylaxis ${ }^{11}$. Therefore, effective therapy directed to prevent or reverse over-inflammatory response, oxidative stress and intestinal barrier damage will be critical and ideal for the development of new treatment strategies of SAP and improve its prognosis.

Ethyl pyruvate (EP), a simple derivative of pyruvic acid, is also an reactive oxygen species (ROS) scavenger, but seems to exert pharmacological effects, such as suppression of inflammation, distinct from those exerted by pyruvate anion. Treatment with EP has been shown to improve survival and/or ameliorate organ dysfunction in a wide variety of pre-clinical models of acute illnesses, such as severe sepsis, acute pancreatitis and stroke ${ }^{12-14}$. Therefore, we reasoned that EP might also be protective against ALI, acting on inhibition of early and late inflammatory cytokines. The present study was designed to evaluate whether EP could be beneficial in a rat model of severe acute pancreatitis ALI.

\section{Methods}

\section{Experimental protocol}

The experimental protocol was approved by the Ethics Committee. All animals received care in accordance with the criteria outlined in the Guide for the Care and Use of Laboratory Animals.

For each experiment, a $28 \mathrm{mM}$ solution of ethyl pyruvate was freshly prepared by dissolving the compound in commercially available Ringer's lactate solution (RLS). This solution was called Ringer's ethyl pyruvate solution (REPS). Commercially available RLS was used as the control solution. All chemicals were purchased from Sigma- Aldrich (St. Louis, MO).

Forty male Wistar rats, weighing 270-330g, were housed in individual cages. Room temperature was kept at $21-22^{\circ} \mathrm{C}$, a 12 hour light-dark cycle was maintained, and all rats were fed standard rat chow and water ad libitum. They were fasted overnight before surgery.

\section{Induction of $A P$}

The rats were operated on under aseptic conditions, and were anesthetized with subcutaneous ketamine $(10 \mathrm{mg} / \mathrm{kg})$ and xylazine $(8 \mathrm{mg} / \mathrm{kg})$. The pancreas was exteriorized through a midline abdominal incision, the proximal bile duct was clamped at the level of the liver hilum, and the distal bile duct was cannulated using a 19-gauge polyethylene catheter through the duodenal wall (Figure 1). AP was induced by intraductal retrograde injection of $0.5 \mathrm{ml} \mathrm{2.5 \%}$ sodium taurocholate (Sigma- Chemical Company TM, St. Louis, Mo., USA), under pressure control. Injection flow was $0.2 \mathrm{ml} / \mathrm{min}$. Previous studies in our laboratory with this model have shown a mortality rate of $35 \%$ after $72 \mathrm{~h}^{10,15}$. After making sure that there was no active bleeding in the abdominal cavity, the abdomen was closed in layers. 
Ethyl-pyruvate reduces lung injury matrix metalloproteinases and cytokines and improves survival in experimental model of severe acute pancreatitis

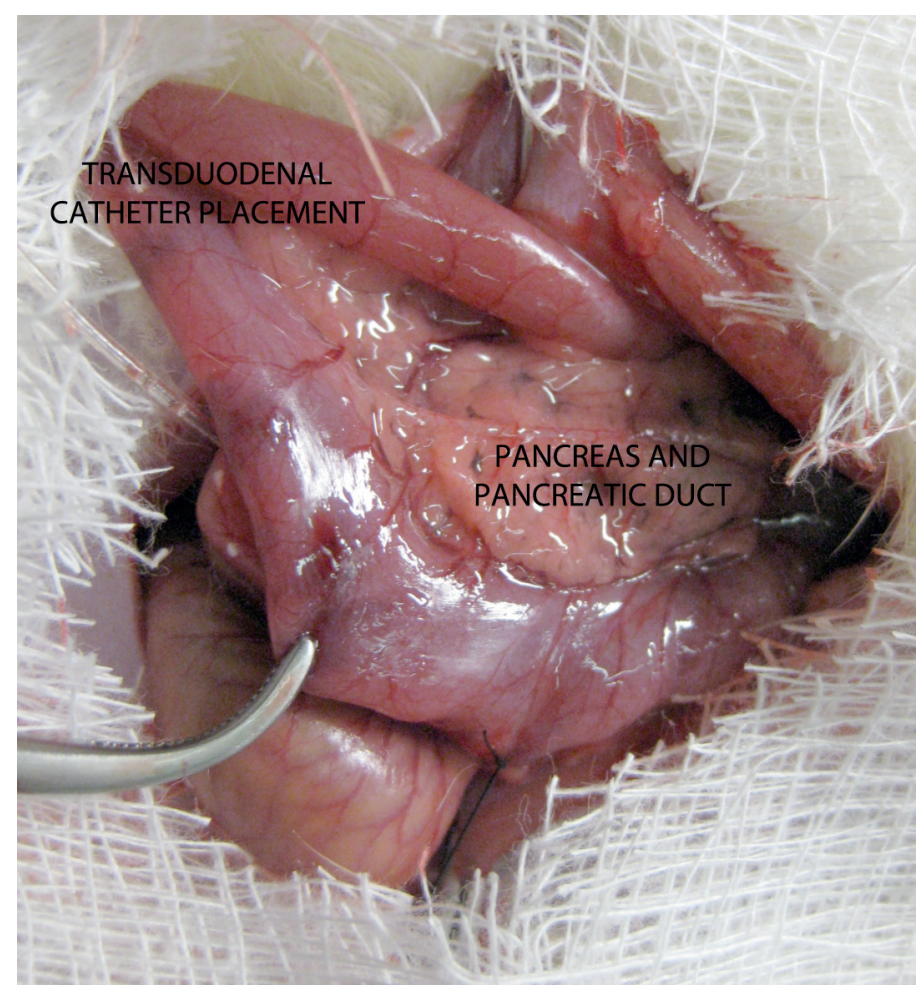

FIGURE 1 - Catheter positioned transduodenally, into the pancreatic duct of the rat, for infusion of the taurocholate $2.5 \%$ and induction of a necrotizing pancreatitis.

\section{Study design}

The animals were randomized into the following 2 experimental groups. Treatment was provided after three and six hours after AP induction. Rats of CT-group were controls and were given intraperitoneal injections of Ringer solution treatment. Those in EP-group were submitted to intraperitoneal Ringer ethylpyruvate solution treatment $(50 \mathrm{mg} / \mathrm{kg})$. The volume of Ringer infused in CT group was calculated to present the same amount as EP group. No antibiotics were administered before, during, or after the procedure. The rats returned to their cages with free access to water after surgery. Two hours later, all animals were again anesthetized and underwent laparotomy and thoracotomy. The thorax was opened, the left atrium was incised, and the lung was perfused in situ with PBS via the pulmonary artery. The flushed lungs were removed immediately, frozen in liquid nitrogen, and stored at $-80^{\circ} \mathrm{C}$ until use. The animals were then, euthanized with ketamine overdose.

In order to study acute lung injury, lung tissue specimen were taken for study of the following parameters: interleukin1B (IL-1), interleukin-6 (IL-6), interleukin-10 (IL-10), tumoral necrosis factor (TNF-a), myeloperoxidase (MPO), maleicdialdehyde (MDA), metalloproteinase 2 e 9, nitric oxide and heat shock protein 70 (HSP70).

\section{Survival protocol}

The second experiment was designed to determine the effect on rat survival of treatment with EP solution $(50 \mathrm{mg} /$ $\mathrm{kg}$ ). Severe acute pancreatitis was induced the same way and the animals were given endovenous fluid resuscitation treatment in groups CT and EP. After surgery, rats were housed in a dry and clean room at a temperature of $20-26^{\circ} \mathrm{C}$, and $12 \mathrm{~h}$ later standard chow and water were freely available. These animals were not reoperated and were kept to record mortality up to ten days after the procedure. Mortality was observed and recorded every day.

\section{Lung tissue preparation}

Frozen tissue $(100 \mathrm{mg})$ was pulverized in liquid nitrogen with a mortar and pestle. Samples were homogenized in radioimmunoprecipitation assay buffer containing $10 \mathrm{mmol} / \mathrm{L}$ of Tris- $\mathrm{HCl}$ ( $\mathrm{pH} 7.5$ ), $1 \%$ of Tergitol, $0.1 \%$ of sodium dodecyl sulfate (SDS), $1 \%$ of sodium deoxycholate, $150 \mathrm{mmol} / \mathrm{L}$ of $\mathrm{NaCl}$, and proteolytic enzyme inhibitors $(40 \mathrm{Kg} / \mathrm{mL}$ of phenylmethylsufonylfluoride and $10 \mathrm{Kg} / \mathrm{mL}$ of pepstatin; Sigma). After debris separation by centrifugation for 45 minutes at $14.000 \mathrm{~g}$, the supernatants were collected and protein concentration was determined by the Bradford method (Bio-Rad Laboratories, Hercules, Calif ). Samples were stored at j80-C until assayed.

Neutrophil Infiltration Myeloperoxidase (MPO) activity was used as a marker for tissue PMN infiltration. Lung tissue fragments $(50 \mathrm{mg})$ were rinsed with saline and homogenized in 0.1 $\mathrm{mmol} / \mathrm{L}$ of sodium phosphate buffer $(80 \mathrm{mmol} / \mathrm{L}$ of NaH2PO4$\mathrm{H} 2 \mathrm{O}$ and $80 \mathrm{mmol} / \mathrm{L}$ of Na2HPO4-7H2O, pH 5.5) containing $0.5 \%$ of hexadecyl trimethyl ammonium bromide and $10 \mathrm{mmol} / \mathrm{L}$ of $3 \mathrm{~N}$ morpholinic) propanesulfonic acid. Suspensions were then centrifuged at $14.000 \mathrm{~g}$ for 30 minutes, and the resulting supernatant was collected. Lung MPO activity was assayed in the supernatant with a mixture of $20 \mathrm{mcL}$ of the sample and $140 \mathrm{KL}$ of $0.1-\mathrm{mmol} / \mathrm{L}$ sodium phosphate buffer ( $\mathrm{pH}$ 5.5). The reaction was started by adding $20 \mathrm{KL}$ of 3,5-tetramethylbenzidine liquid substrate system (Sigma) and $20 \mathrm{KL}$ of $3 \%$ hydrogen peroxide, and the samples were incubated for five minutes. The reaction was stopped by adding $50 \mathrm{KL}$ of $2 \mathrm{~N} \mathrm{H} 2 \mathrm{SO} 4^{17,18}$. The optical density (OD) was read at $450 \mathrm{~nm}$ in a Tecan GENius plate reader (Tecan, Salzburg, Austria). 


\section{Gelatin zymography}

Supernatants from tissue preparation were diluted to a protein concentration of $1 \mathrm{mg} / \mathrm{mL}$. Twenty-microliter aliquots were applied to $10 \%$ polyacrylamide gels with $1 \%$ gelatin incorporated as a substrate for gelatinolytic proteases. After running the gel, the SDS was removed by washing the gel twice in $2.5 \%$ (vol/ vol) Triton X-100 (Sigma) for 30 minutes. The gel was incubated overnight in zymography development buffer containing 50 $\mathrm{mmol} / \mathrm{L}$ of Tris- $\mathrm{HCl}(\mathrm{pH} 7.4), 2 \mathrm{mmol} / \mathrm{L}$ of $\mathrm{NaN} 3$, and $5 \mathrm{mmol} / \mathrm{L}$ of $\mathrm{CaCl}$. We added MMP inhibitors $(10 \mathrm{mmol} / \mathrm{L})$ of EDTA and $10 \mathrm{mmol} / \mathrm{L}$ of 1,10 -phenatroline) to the development buffer to assess whether the observed gelatinolytic bandswere caused by serine protease activity or MMP activity. After development, the gels were stained for 3 hours in 45\% methanol/ 10\% glacial acetic acid containing 1\% (wt/vol) Comassie Blue R-250 (Bio-Rad Laboratories, Hercules, Calif ) and were subsequently partially destained with the same solution without dye. The gelatinolytic activity of each MMP was qualitatively evaluated as a clear band against the blue-stained gelatin background ${ }^{5,17}$. Band intensity was quantitated using the software Image J (Research Services Branch, National Institutes of Health, Bethesda, Md).

\section{Immunoblotting}

Protein expression was performed using an SDSY polyacrylamide gel electrophoresis under reducing conditions. Lung tissue extracts $(25 \mathrm{Y} 100 \mathrm{Kg} / \mathrm{mL})$ were boiled in equal volumes of loading buffer $(150 \mathrm{mmol} / \mathrm{L}$ of Tris- $\mathrm{HCl}, \mathrm{pH} 6.8 ; 4 \%$ of SDS; $20 \%$ of glycerol; $15 \%$ of A-mercaptoethanol; and $0.01 \%$ of bromophenol blue) and were subjected to electrophoresis in $10 \%$ polyacrilamide gels. After electrophoretic separation, proteins were transferred to Hybond-P membranes (Amersham Pharmacia Biotech, Buckinghamshire, England). Membranes were blocked with $5 \%$ nonfat dry milk in Tris-buffered saline and $0.5 \%$ Tween (TBST) for 1 hour. Primary antibodies against HSPs (polyclonal goat antirat, 1:1000; Santa Cruz Biotechnology, Inc, Santa Cruz, Calif) were used. Antibodies were incubated at 4-C overnight. After washing twice with TBST, secondary antibody horseradish peroxidase conjugate (Santa Cruz Biotechnology, Inc) was applied at a dilution of 1:1000 for two hours. Blots were washed in TBST twice for 30 minutes, incubated in enhanced chemiluminescence reagents (SuperSignal detection kit; Pierce, Rockford, Ill), and exposed to Kodak O-OMAT-AR photographic film (Kodak, Rochester, NY). Band intensity was quantitated using the software Image J (Research Services Branch, National Institutes of Health).

\section{Cytokine measurements}

The concentrations of TNF-a, IL-6 and IL-1b were measured using commercially available enzyme-linked immunosorbent assay kits (R\&D Systems, Minneapolis, MN) according to the manufacturer's instructions. The sensitivities for TNF-a, IL-6 and IL-1b were $15 \mathrm{pg} / \mathrm{ml}, 5 \mathrm{pg} / \mathrm{ml}$ and $5 \mathrm{pg} / \mathrm{ml}$, respectively.

$M D A$

Evaluation of maleic dialdehyde activity was performed with samples of pulmonary parenchyma that were collected, rinsed in saline buffer, snap-frozen in liquid nitrogen and stored at $-80^{\circ} \mathrm{C}$, until analyzed. MDA contents of the lung were tested by enzymatic-spectrophotometric kit (Jian Cheng Biotech Ltd, China). The tissue levels of MDA were interpreted as an indicator of lipid peroxidation. All these indexes were examined according to the manufacturer's instructions.

\section{Statistical analysis}

Overall survival was calculated by the Kaplan-Meier Estimate. The log-rank test and the Chi-square test were used to compare survival curves in the two groups. A p value $<0.05$ was considered statistically significant.

Data were expressed as mean (SEM). Comparisons between experimental groups were performed by analysis of variance, and the $t$ Student test was used as posttest to compare individual group. All analyses were performed using SPSS (Statistical Package for the Social Science) 13.0 for Windows.

\section{Results}

\section{Macroscopic findings}

Autopsies were carried out on dead rats. In all cases, much yellow or bloody exudate with a slight smell would come out of the peritoneal cavity when the abdomen was opened. The gastrointestinal tract was obviously ectatic and edematous. The intestine would expand to 2-3 times of normal with gas and fluid inside and with a thinner wall. The pancreas was swollen and dark colored with some local hemorrhagic spots (Figure 2). Yellowwhite saponified spots were always found in the peripancreatic omentum and retroperitoneum. The lower part of the spleen was always dark black. A pancreatic or hepatic abscess could be 
observed if the rat died after the 4th day or later. Compared with SAP group, the severity of macroscopic changes was remarkably attenuated in the EP group.

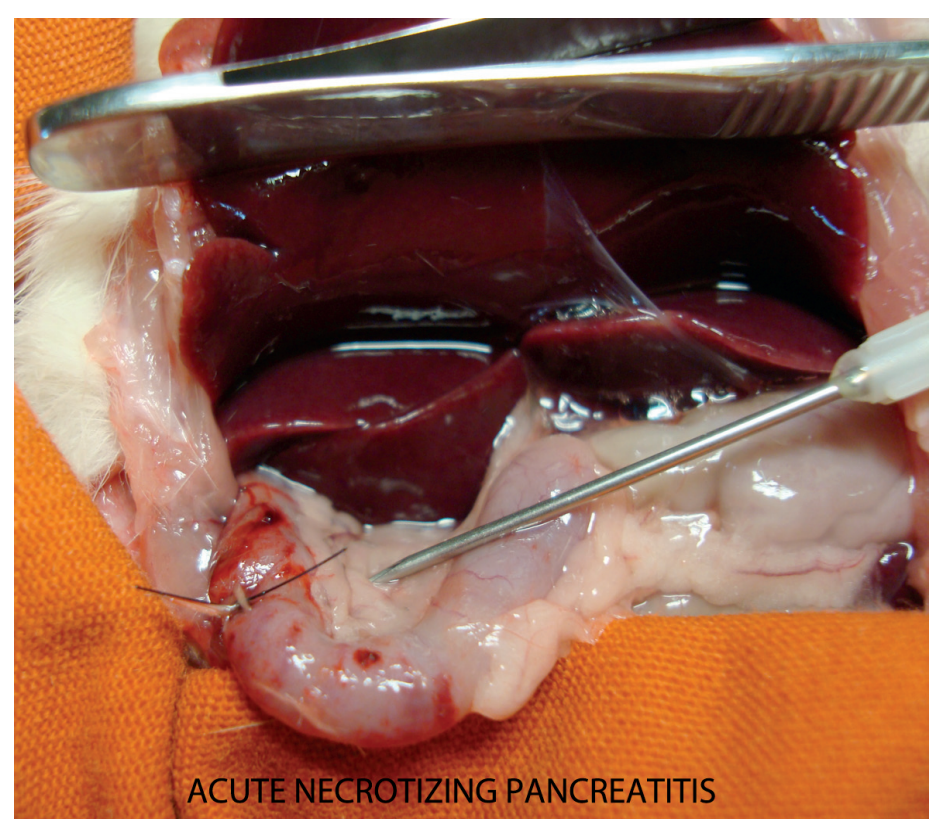

FIGURE 2 - At re-laparotomy, the lung was resected and freezed. The pancreas was swollen and dark colored with some local hemorrhagic spots, revealing an acute necrotizing pancreatitis, induced by the artificial bile, taurocholate $2.5 \%$.

Ringer ethyl-pyruvate solution reduces interleukin-6 in the lungs

The Table 1 shows the IL-6 measurement following the Ringer-EP treatment and the Ringer (CT group).

TABLE 1 - IL-6 values found in pulmonary specimen, with comparison between the two groups.

\begin{tabular}{cccccc}
\hline Group & Media & $\begin{array}{c}\text { Standard } \\
\text { deviation }\end{array}$ & Median & Minimum & Maximum \\
\hline CT & 57.18 & 38.88 & 45.65 & 20.45 & 155.25 \\
\hline EP & 28.54 & 11.51 & 29.48 & 12.39 & 49.90 \\
\hline
\end{tabular}

As it can be seen in Figure 3, the levels of IL-6 were significantly lower in the EP group, when compared to the CT group. $\mathrm{t}(18)=2.23 ; 95 \% \mathrm{IC}=1.70$ a $55.58 ; \mathrm{p}=0.038$.

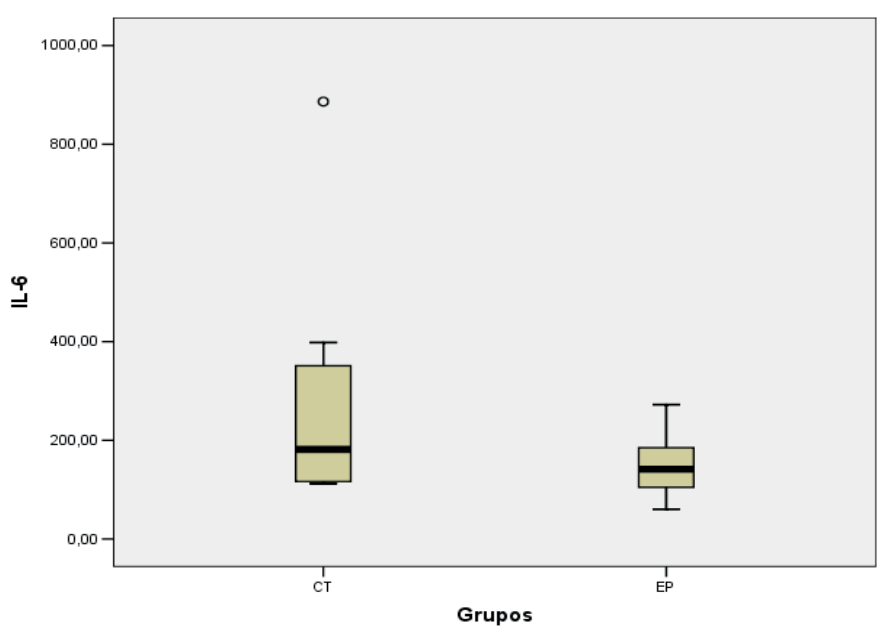

FIGURE 3 - Boxplot of IL-6 measurement on pulmonary specimen in a comparison between groups $\mathrm{CT}$ and $\mathrm{EP}$.

Ringer ethyl-pyruvate solution diminishes MMP-2 in the Lungs

The MMPs are released upon neutrophil degranulation and aid in basement membrane digestion and neutrophil sequestration in the lung. Pulmonary MMP-2 activity demonstrated after AP was markedly high when compared to the CT group at eight hours.

There was significant difference in the serum gelatinase profiles of the two experimental groups. The Table 2 shows the MMP-2 measurement following the Ringer-EP treatment and the Ringer (CT group)

TABLE 2 - Difference of MMP-2 measurement between group EP and CT.

\begin{tabular}{cccccc}
\hline Group & Media & $\begin{array}{c}\text { Standard } \\
\text { deviation }\end{array}$ & Median & Minimum & Maximum \\
\hline CT & 25278.80 & 3086.35 & 26087.00 & 20183.00 & 28528.00 \\
\hline EP & 16998.80 & 2238.73 & 17100.00 & 13493.00 & 19184.00 \\
\hline
\end{tabular}

As it can be seen in Figure 4, the levels of MMP-2 were significantly lower in the EP group, when compared to the CT group. $\mathrm{t}(8)=4.86 ; 95 \% \mathrm{IC}=4347.95$ a $12212.05 ; \mathrm{p}=0.001$. 


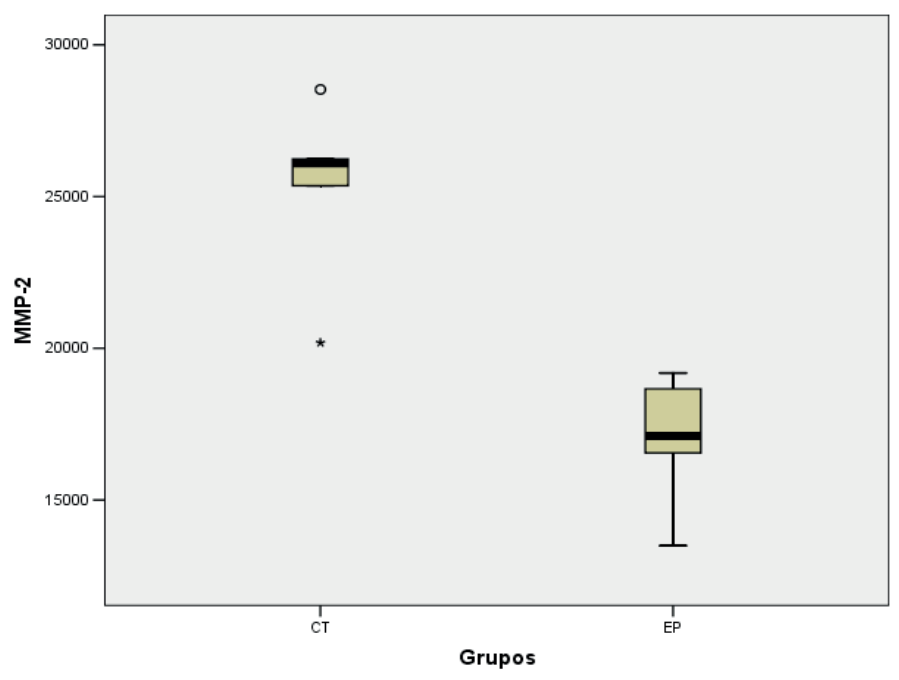

FIGURE 4 - Boxplot of MMP-2 expression comparing both groups (CT and EP).

Figure 5depicts thereductioninmatrixmetalloproteinase-2 expression, following EP treatment. No reduction was observed in the control group.

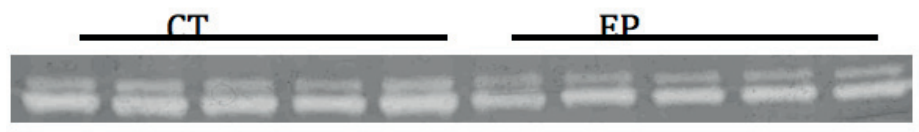

FIGURE 5 - Gelatin zymography was used to assess the effects of EP on the MMP-2 activity in the lung tissue. MMP-2 activity significantly decreased after eight hours, in the group treated by Ringer-EP solution $(\mathrm{p}=0.001)$.

As shown in Tables 3 and 4, no differences were found in IL-1B, IL-10, TNF, NO, MPO, MDA and MMP-9. Furthermore, EP group animals had levels of HSP70 activity comparable to that of the control. Nevertheless, the significant differences between groups concerning IL-6 and MMP-2 show an important role of the EP in inflammatory response.

TABLE 3 - Biochemical parameters analysis subsequent to Ringer-EP and Ringer solution treatment.

\begin{tabular}{|c|c|c|c|c|c|c|}
\hline Parameters & $\mathbf{N}$ & Media & SD & Median & Minimum & Maximum \\
\hline \multicolumn{7}{|l|}{ CT-group } \\
\hline IL-1 & 10 & 86.50 & 95.18 & 48.99 & 29.04 & 348.19 \\
\hline IL-6 & 10 & 57.18 & 38.88 & 45.65 & 20.45 & 155.25 \\
\hline IL-10 & 10 & 265.70 & 240.02 & 181.32 & 112.49 & 886.29 \\
\hline MDA & 10 & 0.02 & 0.02 & 0.02 & 0.00 & 0.07 \\
\hline $\mathrm{NO}$ & 10 & 2.22 & 1.88 & 1.69 & 0.38 & 5.83 \\
\hline MPO & 10 & 1.89 & 3.00 & 0.78 & 0.25 & 10.07 \\
\hline TNF & 10 & 67.10 & 79.61 & 33.64 & 24.05 & 283.83 \\
\hline MMP-2 & 5 & 25278.80 & 3086.35 & 26087.00 & 20183.00 & 28528.00 \\
\hline MMP-9 & 5 & 21345.80 & 4021.21 & 19566.00 & 17105.00 & 26120.00 \\
\hline HSP 70 & 5 & 21736.40 & 3480.73 & 21326.00 & 18984.00 & 27638.00 \\
\hline \multicolumn{7}{|l|}{ EP - group } \\
\hline IL-1 & 10 & 76.25 & 41.01 & 63.72 & 46.29 & 187.62 \\
\hline IL-6 & 10 & 28.54 & 11.51 & 29.48 & 12.39 & 49.90 \\
\hline IL-10 & 10 & 148.70 & 63.23 & 141.77 & 60.75 & 272.46 \\
\hline MDA & 10 & 0.01 & 0.01 & 0.01 & 0.01 & 0.03 \\
\hline Nitrito & 10 & 4.94 & 4.36 & 4.56 & 0.52 & 12.35 \\
\hline MPO & 10 & 0.48 & 0.43 & 0.27 & 0.07 & 1.22 \\
\hline TNF & 10 & 31.95 & 10.91 & 28.27 & 21.22 & 56.70 \\
\hline MMP-2 & 5 & 16998.80 & 2238.73 & 17100.00 & 13493.00 & 19184.00 \\
\hline MMP-9 & 5 & 18760.60 & 8179.36 & 20477.00 & 9243.00 & 29200.00 \\
\hline HSP 70 & 5 & 22959.20 & 3233.67 & 22125.00 & 19778.00 & 28414.00 \\
\hline
\end{tabular}


TABLE 4 - t Student test of independent specimen, comparing biochemical data, between groups.

\begin{tabular}{|c|c|c|c|c|c|c|}
\hline \multirow{2}{*}{$\frac{\text { Parameters }}{\text { IL-1 }}$} & \multirow{2}{*}{$\begin{array}{c}\mathbf{T} \\
0.31\end{array}$} & \multirow{2}{*}{$\begin{array}{c}\text { DF } \\
18\end{array}$} & \multicolumn{3}{|c|}{ 95\% IC (difference) } & \multirow{2}{*}{$\begin{array}{c}\mathbf{P} \\
0.758\end{array}$} \\
\hline & & & -58.61 & $\mathrm{a}$ & 79.11 & \\
\hline IL-6 & 2.23 & 18 & 1.70 & a & 55.58 & $0.038^{*}$ \\
\hline IL-10 & 1.49 & 18 & -47.91 & a & 281.90 & 0.153 \\
\hline MDA & 1.31 & 18 & 0.00 & $\mathrm{a}$ & 0.02 & 0.206 \\
\hline Nitrito & -1.81 & 18 & -5.87 & $\mathrm{a}$ & 0.44 & 0.088 \\
\hline MPO & 1.47 & 18 & -0.61 & a & 3.41 & 0.160 \\
\hline TNF & 1.38 & 18 & -18.23 & $\mathrm{a}$ & 88.54 & 0.183 \\
\hline MMP-2 & 4.86 & 8 & 4347.95 & a & 12212.05 & $0.001 *$ \\
\hline MMP-9 & 0.63 & 8 & -6814.25 & a & 11984.65 & 0.544 \\
\hline HSP 70 & -0.58 & 8 & -6122.40 & $\mathrm{a}$ & 3676.80 & 0.581 \\
\hline
\end{tabular}

Ethyl pyruvate improves survival in rats with acute pancreatitis

As expected, all ten of the rats in the control group died until the third day of severe AP induction (Figure 6). In contrast, in the group with pancreatitis treated with Ringer-EP, only one of ten animals $(10 \%)$ reached the tenth days of this period of observation. Seven of ten $(70 \%)$ rats in the EP group survived after the third day. Survival time was significantly longer in the EP group than the CT group ( $\mathrm{p}=0.011)$.

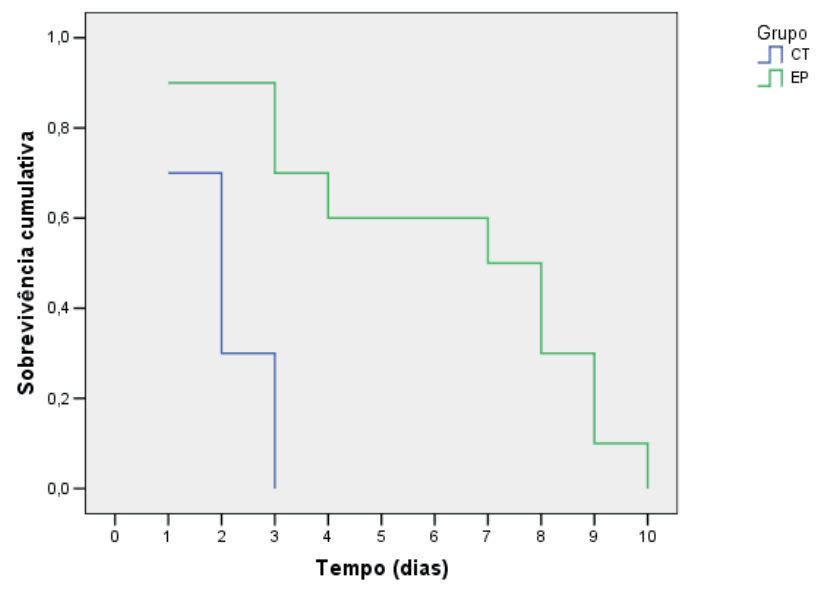

FIGURE 6 - Kaplan-Meier survival curves of SAP rats. 1: Control group; 2: EP treatment group. Administration of EP dose prevents the lethality of ALI. Survival curves $(50 \mathrm{mg} / \mathrm{kg} / \mathrm{d})$ of EP versus survival in vehicletreated controls $(\mathrm{CT}) ; \mathrm{p}=0.01$ by log-rank test.

Table 5 details de survival findings after statistical evaluation. Animals from the EP group had improved survival after necrotizing acute pancreatitis induction.
TABLE 5 - Comparison of Kaplan-Meier survival curve, after ten days.

\begin{tabular}{ccccc}
\hline Group & $\begin{array}{c}\text { Frequency } \\
(\%)\end{array}$ & $\mathbf{X}^{2}$ & GL & $\mathbf{P}$ \\
\hline CT & 100 & 11.23 & 1 & $0.001^{*}$ \\
EP & 100 & & & \\
\hline
\end{tabular}

\section{Discussion}

The pathophysiological events in experimental acute pancreatitis consists of activation of pancreatic enzymes within acinar cells, the release of these activated enzymes in the interstitial, the autodigestion of the pancreas, and the release of activated pancreatic enzymes and other factors into the circulation that result in the development of multiple organ dysfunction. Currently, the factors that determine the ultimate severity of the acute pancreatitis remain uncertain ${ }^{16}$.

ALI is the most common and severe complication of SAP during the early stage. About one third of patients with SAP may develop acute lung injury, which is manifested clinically as acute respiratory distress syndrome (ARDS). During the first week, approximately $20 \%$ patients with acute lung injury develop ARDS. It is the first cause of patients' death during the early stage ${ }^{17}$.

The initial clinical response to pancreatitis is a systemic inflammatory response (SIRS), which, if abnormally persistent, develops into a worsening scenario of tissue damage and sepsis resulting in multiple organ dysfunction syndrome (MODS) $)^{18-20}$.

These pathological changes in the pancreatic gland are responsible for releasing active pancreatic enzymes into the bloodstream and stimulating the production of inflammatory cytokines such as interleukin-1, interleukin-6 and interleukin-8 from neutrophils, macrophages and lymphocytes. The release 
of those interleukins and tumor necrosis factor- $\alpha$ (TNF- $\alpha$ ) from macrophages triggers an inflammatory cascade, which leads to the systemic inflammatory response syndrome (SIRS) ${ }^{11}$.

The critical players of this interaction include the proinflammatory cytokines IL-1beta, TNF-alpha, IL-6, interleukin-10 (IL-10), neutrophils infiltration, lipidic peroxidation metalloproteinase 2 e 9, nitric oxide and heat shock protein 70 $(\mathrm{HSP} 70)^{10}$.

Despite several experimental models of severe acute pancreatitis have been reported, one of the best-standardized compounds to use is sodium taurocholate. Infusion of $0.2 \mathrm{ml} / \mathrm{kg}$ of $3 \%, 4.5 \%$ or $5 \%$ solution induced acute haemorrhagic pancreatitis with $72-\mathrm{h}$ mortality rates of $24 \%, 71 \%$ and $100 \%$, respectively. This model is appropriate for studies of systemic issues.

EP administration significantly improves the survival of lethal hemorrhagic shock in standard models, and significantly inhibits the systemic release of both early (TNF- $\alpha$ ) and late (HMGB1) cytokines that mediate the lethality of sepsis and systemic inflammation, even when administered $24 \mathrm{~h}$ after cecal puncture.

The pharmacologic mechanisms responsible for the antiinflammatory effects of ethyl pyruvate remain poorly understood. It is known, however, that pyruvate reacts non-enzymatically to scavenge the reactive oxygen species (ROS) hydrogen peroxide. Thus, it is conceivable that the beneficial effects of ethyl pyruvate are due to its properties as an antioxidant. Interestingly, however, recent observations from our laboratory suggest that ROS scavenging is unlikely to be the entire explanation for the antiinflammatory properties of ethyl pyruvate. The reduction on MMP-2 levels may lead to another role of the EP in ameliorating the inflammatory cascade of severe AP.

\section{Cytokines}

It is likely that pro-inflammatory cytokines, notably TNF-a, IL-1b and IL-6, participate in the early development of inflammation; they have been shown to play a crucial role in ALI and ARDS. Since cytokines could play an important role in AP, many agents have been used in experimental animals to ameliorate or prevent the inappropriate activation of the immune systems.

In our study, IL-6 were significantly reduced in the EP group. However, the other cytokines presented no significant alterations throughout the research. The following results were found in the measurements of IL-1B, IL-10 and TNF-a, respectively: $p=0.758, p=0.153$ and $p=0.183$.

IL-6 levels are elevated in pancreatitis and serve as markers of severity of pancreatitis, in addition to paralleling the course of the disease. IL-6 levels can also help to predict the possibility of pulmonary complications. In this aspect, IL-6 is superior to C-Reactive protein or B-2 microglobulin and has been shown to rise $12-24 \mathrm{~h}$ before other inflammatory markers.

\section{Matrix metalloproteinases}

The matrix metalloproteinases (MMPs) are believed to be the main physiological mediators of ECM degradation. In adult tissue, low level MMP expression mediates normal matrix remodeling, while during inflammation and injury, large amounts of MMPs are produced presumably to repair damaged ECM. By cleaving ECM components, MMPs could play a role in the repair of the alveolar epithelium during acute lung injury. Gelatinases, MMP-2 and MMP-9, are known to degrade almost all basement membrane constituents, including type IV collagen, nidogen and laminin, and gelatins.

Matrix metalloproteinases 2 and 9 are important mediators in tissue inflammatory response and have also been implicated in lung injury ${ }^{8}$. Elevated MMP activity in this study in demonstrated by gelatin zymography and confirmed by the fluorogenic substrate. Extracellular matrix degradation from MMP-2 and MMP-9 may result in capillary leak and play a causative role in the lung injury.

In acute pancreatitis, the role of polymorphonuclear neutrophils (PMNs) for alveolar membrane dysfunction and lung injury has been clarified before. MMP-2 is synthesized by a wide variety of cells, including fibroblasts, endothelial cells and alveolar epithelial cells. Elevated levels of active MMP-2 have been shown to be a specific pulmonary response during ARDS, independent of other organ failures. Our findings of a significant reduction in MMP-2 in the EP group may explain the survival improvement observed during the second experiment. We cannot explain the reason for the not altered measurement of MMP-9, indeed.

Although the manipulations of specific mediators in animal models may be promising, they may not transition well to the human clinical setting. However, continued reliance on experimental animal models of acute pancreatitis may be necessary to determine the underlying causes of disease. Measurement of the inflammatory response may also serve to identify diagnostic markers for the presence of acute pancreatitis and provide insight into prognosis. Understanding the models, documenting the markers, and deciphering the mediators have the potential to improve treatment of acute pancreatitis.

Our data showed that administration of EP even when initiated $3 \mathrm{~h}$ after induction of ALI significantly increased survival. 
The levels of the IL-6 had decreased significantly. The expression of MMP-2 was also significantly affected; indicating that EP modulation of inflammatory cytokines contributes to the beneficial effects on survival.

\section{Conclusions}

Ethyl-pyruvate (EP) treatment in established severe acute pancreatitis (SAP) lung injury reduced the severity of AP and the mortality rate in an animal model of SAP. These findings suggest that EP strategies are of potential therapeutic value in the treatment of associated lung injury (ALI) in severe AP.

\section{References}

1. McKay CJ, Imrie CW. The continuing challenge of early mortality in acute pancreatitis. Br J Surg. 2004;91:1243-4.

2. Gloor B, Müller CA, Worni M, Martignoni ME, Uhl W, Büchler MW. Late mortality in patients with severe acute pancreatitis. Br J Surg. 2001;88:975-9.

3. Chen YP, Ning JW, Ji F. Establishment of the critical period of severe acute pancreatitis-associated lung injury. Hepatobiliary Pancreat Dis Int. 2009;8(5):535-40.

4. Makhija R, Kingsnorth AN. Cytokine storm in acute pancreatitis. J Hepatobiliary Pancreat Surg. 2002;9:401-10.

5. Pereda J, Sabater L, Cassinello N, Gómez-Cambronero L, Closa D, Folch-Puy E, Aparisi L, Calvete J, Cerdá M, Lledó S, Viña J, Sastre J. Effect of simultaneous inhibition of TNF-alpha production and xanthine oxidase in experimental acute pancreatitis: the role of mitogen activated protein kinases. Ann Surg. 2004;240:108-16.

6. Gomez-Cambronero LG, Sabater L, Pereda J, Cassinello N, Camps B, Viña J, Sastre J. Role of cytokines and oxidative stress in the pathophysiology of acute pancreatitis: therapeutical implications. Curr Drug Targets Inflamm Allergy. 2002;1:393-403.

7. Okamoto T, Akuta T, Tamura F, van Der Vliet A, Akaike T. Molecular mechanism for activation and regulation of matrix metalloproteinases during bacterial infections and respiratory inflammation. Biol Chem. 2004;385(11):997-1006.

8. Muhs BE, Patel S, Yee H, Yee H, Marcus S, Shamamian P. Inhibition of matrix metalloproteinases reduces local and distant organ injury following experimental acute pancreatitis. J Surg Res. 2003;109(2):110-7.

9. Steinberg J, Halter J, Schiller HJ, Dasilva M, Landas S, Gatto LA, Maisi P, Sorsa T, Rajamaki M, Lee HM, Nieman GF. Metalloproteinase inhibition reduces lung injury and improves survival after cecal ligation and puncture in rats. J Surg Res. 2003;111(2):185-95.

10. Moretti AI, Rios EC, Soriano FG, de Souza HP, Abatepaulo F, Barbeiro DF, Velasco IT. Acute pancreatitis: hypertonic saline solution increases heat shock proteins 70 and 90 and reduces neutrophil infiltration in lung injury. Pancreas. 2009;38(5):507-14.

11. Tonsi AF, Bacchion M, Crippa S, Malleo G, Bassi C. Acute pancreatitis at the beginning of the 21st century: the state of the art. World J Gastroenterol. 2009;15:2945-59.

12. Kao KK, Fink MP. The biochemical basis for the anti-inflammatory and cytoprotective actions of ethyl pyruvate and related compounds. Biochem Pharmacol. 2010;80(2):151-9.

13. Fink MP. Ethyl pyruvate: a novel anti-inflammatory agent. J Intern
Med. 2007;261(4):349-62.

14. Zingarelli B. Ethyl pyruvate: a simple solution? Crit Care Med. 2004;32(7):1603-4.

15. Machado MC, Coelho AM, Pontieri V, Sampietre SN, Molan NA, Soriano F, Matheus AS, Patzina RA, Cunha JE, Velasco IT. Local and systemic effects of hypertonic solution $(\mathrm{NaCl} 7.5 \%)$ in experimental acute pancreatitis. Pancreas. 2006;32:80-6.

16. Su KH, Cuthbertson C, Christophi C. Review of experimental animal models of acute pancreatitis. HPB (Oxford). 2006;8:264-86.

17. Kan SH, Huang F, Tang J, Gao Y, Yu CL. Role of intrapulmonary expression of inducible nitric oxide synthase gene and nuclear factor kappaB activation in severe pancreatitis-associated lung injury. Inflammation. 2010;33(5):287-94

18. Davies MG, Hagen PO. Systemic inflammatory response syndrome. Br J Surg. 1997;84:920-35.

19. Ware LB, Matthay MA. The acute respiratory distress syndrome. N Engl J Med. 2000;342:1334-49.

20. Malangoni MA, Martin AS. Outcome of severe acute pancreatitis. Am J Surg. 2005;189:273-7.

\section{Acknowledgments}

To Postgraduate Program in Gastrointestinal Division, Federal University of Sao Paulo, Prof. Dr. Heraldo Possolo de Souza and Prof. Dr. Irineu Tadeu Velasco for technical assistance and advice on the Emergency Medicine Lab, LIM 51, USP, Brazil.

\section{Correspondence:}

Dr. Jacques Matone

Avenida Angélica, 2503/66

01227-200 São Paulo - SP Brasil

Tel.: (55 11)3257-2398

Fax: $(55$ 11)3237-4818

matone@einstein.br

Received: April 18, 2013

Review: June 20, 2013

Accepted: July 22, 2013

Conflict of interest: none

Financial sources: The research was financed by the Postgraduate Program in Gastrointestinal Surgery (UNIFESP-EPM) and Emergency Medicine Lab - LIM51 (USP).

${ }^{1}$ Research performed at Experimental Laboratory of Emergency Medicine, LIM51, University of Sao Paulo (USP), Brazil. Part of PhD degree thesis, Postgraduate Program in Gastrointestinal Surgery, Federal University of Sao Paulo - Paulista Medical School (UNIFESP-EPM), Sao Paulo, Brazil. Tutor: Prof. Alberto Goldenberg. 